\title{
First-principles study on the adsorption structure of water molecules on a pyrite (100) surface
}

\author{
Yingchao Liu 1, Jianhua Chen 2, Yuqiong Li 2, Junjie Zhang 2, Duan Kang ${ }^{3}$ \\ ${ }^{1}$ School of Chemistry and Chemical Engineering, Guangxi University, Nanning, 530004, China \\ 2 School of Resources, Environment and Materials, Guangxi University, Nanning, 530004, China \\ ${ }^{3}$ Research Institute of Petroleum Exploration and Development, PetroChina, Beijing, 100083, China \\ Corresponding author: yql@gxu.edu.cn (Y. Li)
}

\begin{abstract}
The hydration structure of water molecule adsorption at different coverages of a monolayer on a pyrite (100) surface were simulated using the density functional theory (DFT) method. The results demonstrate that the Fe-O interaction weakens and the adsorption energy per water molecule decreases with increasing water coverage, except at a monolayer coverage of 12/12 (i.e., full coverage). H-S and $\mathrm{H}-\mathrm{O}$ hydrogen bonds were formed on the nearest surface layer. When large amounts of water molecules adsorb onto the surface, the adsorbed water molecules can be divided into three layers: the layer nearest to the surface, the second nearest to the surface, and the layer farthest from the surface. The thickness of the former two layers is approximately $5.5 \AA$. The three layers have water densities of $1.12 \mathrm{~g} / \mathrm{cm} 3$, $1.08 \mathrm{~g} / \mathrm{cm} 3$, and $0.95 \mathrm{~g} / \mathrm{cm} 3$, respectively, suggesting that there is a strong interaction between the pyrite surface and water molecules and the influence of surface structure on water adsorption reaches a distance of more than $10 \AA$. Dynamics simulations suggest that the water molecules close to the mineral surfaces are in an orderly arrangement while those far from the surface are disordered.
\end{abstract}

Keywords: pyrite, water molecules, adsorption

\section{Introduction}

Pyrite $\left(\mathrm{FeS}_{2}\right)$, one of the most widely existing sulphide minerals on Earth (Tauson et al., 2008), is found to coexist with other sulphide minerals (including $\mathrm{Cu}, \mathrm{Pb}, \mathrm{Zn}$, etc.) and coal. Some studies have indicated that when pyrite is exposed to air and water, the pyrite surface becomes hydrophilic due to oxidation and hydrolysis reactions (Chander, 1991, Xi et al., 2019). In froth flotation, based on the difference in mineral surface hydrophilicity, the hydrophobic minerals can be separated from hydrophilic minerals. Hence, the adsorption of water on the mineral surface is crucial during flotation.

Some experimental and theoretical results indicate that an isolated water molecule is absorbed onto the pyrite surface through interaction between the outermost layer $\mathrm{Fe}$ and $\mathrm{O}$ atoms and the adsorption energy of an isolated water molecule can reach more than $-50 \mathrm{~kJ} / \mathrm{mol}$ (Stirling et al., 2003, Sit et al., 2012, Rosso et al.,1999, Li et al., 2018, Nesbitt and Muir, 1994, Knipe et al., 1995). With an increase in $\mathrm{H}_{2} \mathrm{O}$ dosage, Pettenkofer et al. reported that when the pyrite surface has intermediate coverage (approximately 0.5L), the intensity of the Fe related signal is weaker and the associated $\mathrm{O}$ signal is stronger than on the clean pyrite surface, via LEIS-spectra. For approximately $1 \mathrm{~L}$, only the O-associated signal remains, suggesting that water molecules are preferentially absorbed onto the Fe site (Pettenkofer et al., 1991). During the flotation separation process, the pulp contains excess water molecules and a hydration layer will be formed on the surface and affect the interaction of flotation reagents with the mineral surface to some extent as the hydration layer can hinder the adsorption of flotation reagents or compete with the flotation reagents to adsorb onto the surface (Li et al., 2019). Chen et al. (2014) studied the adsorption states of multi-layer water molecules on galena and pyrite surfaces. It is suggested that there are clear differences in the relaxation and electronic properties after the adsorption of multilayer 
water molecules, which may affect subsequent interfacial reactions. Knipe et al. (1995) utilized X-ray photoelectron spectroscopy (XPS) to analyse the interaction between sulphide surfaces and water vapour for different water contents. They found that the interaction process of pyrrhotite and pyrite with water is different and that water molecules interact with the $\mathrm{Fe} 3 \mathrm{~d}\left(\mathrm{e}_{\mathrm{g}}\right)$ orbital of the pyrite surface. Based on some experiments on fracture surfaces, they proposed that the adsorption degree of water molecules decreases with an increase in the vacuum layer. However, although the adsorption of the multilayer water molecules on the pyrite surface has been intensively studied, based on the binding energy of oxygen derived from XPS, it is suggested that it is difficult to propose a clear mechanism for the interaction between water molecules and the pyrite surface.

The interfacial water at hydrophobic and hydrophilic mineral surfaces has a different structure (Jin, 2016), which is a significant parameter for flotation. Various analytical methods, e.g., XPS (Nesbitt and Muir, 1994, Knipe et al., 1995, Feng et al., 2020, Guevremont et al., 1998), grazing incidence X-ray diffraction (GIXRD) (Sandrina and Uta, 2010), and Fourier transform infrared spectroscopy (FTIR) (Cheng et al., 2013) have been widely used to study the interfacial water structure between water molecules and minerals. Moreover, density functional theory (DFT) and molecular dynamics simulations (MDS) can be adopted to investigate the interfacial water structure at the atomic level (Pettenkofer et al., 1991, Li et al., 2019, Philpott et al., 2004, Jin et al., 2015, Criscenti et al., 2018). Based on molecular dynamics (MD), Philpott et al. (2004) analysed the interaction of the water-iron pyrite $\mathrm{FeS}_{2}$ interface. They suggested that water molecules closest to the pyrite surface are bound by covalent interactions with the iron atoms in the grooves that are parallel to the crystal axis and approximately two atoms wide. They discussed the density of water molecules on the surface of pyrite as a function of distance. In addition, they found that the water molecules bound by iron and sulphur are obviously connected in the hydrogen bond network and the interaction between the first water layer and pyrite surface is stronger at high temperatures. Jin et al. (2015) also utilized MDS to study the interfacial water structure at fresh and oxidized pyrite (100) surfaces. They discussed the influence of pyrite surface properties on interfacial water. However, a detailed understanding of hydration structures on pyrite surfaces is still required.

Therefore, this study focuses on the hydration structure after water molecule adsorption at different coverages, including at $1 / 12,6 / 12$, and $12 / 12$ of a monolayer, and 24/12, 36/12, 72/12, and 120/12 of water in excess of a monolayer on the pyrite (100) surface through the calculation of density functional theory (DFT) based first-principles. Molecular dynamics simulations were performed to investigate the radial distribution function of water molecules on the pyrite surface at different temperatures.

\section{Computational model and methods}

Based on density functional theory (DFT) and the plane wave pseudopotential, geometry optimisation was performed using the CASTEP (Clark et al., 2005, Segall et al., 2002) module. The generalised gradient approximation (GGA) (Perdew 1992 and 1993) was used as the gradient correction approximation and PW91 as the exchange-correlation functional. The DFT-D was adopted for the treatment of the van der Waals force between water molecules. After testing, a plane wave cut-off energy of $350 \mathrm{eV}$ was chosen and the Brillouin zone was sampled with a special Monkhorst-Pack (Chadi, 1977) of a $1 \times 2 \times 1$ grid. Ultra-soft pseudopotentials (Vanderbilt, 1990) were chosen to calculate the interaction between valence electrons. The computed lattice parameters of pyrite are $a=b=c=5.3798$ $\AA$, which is close to the experimental value of $5.4160 \AA$ (Prince et al., 2005). The pyrite (100) surface was cut from an optimized bulk cell and a vacuum layer with $20 \AA$ thickness was built along the z-direction. A $3 \times 2$ supercell in the $x$ - and $y$-directions was fabricated, with 12 Fe sites on the surface, and a slab size with 15 atomic layers was adopted. The Fe atoms on the (100) surface were five-coordinated and the $S$ atoms were three-coordinated. We constructed the adsorption structures of the isolated water molecule (1/12), six water molecules (1/2), twelve water molecules (full adsorption), and several layers of water on the surface. In our simulation, the density of state, charge density analysis, and Mulliken population were calculated for the state of water molecule adsorption on the surface. The calculation parameters were set as follows: $0.002 \AA$ of convergence tolerances for atomic displacement, $0.05 \mathrm{eV} / \AA$ of the maximum force, and $0.1 \mathrm{GPa}$ of the maximum stress. The self-consistent field (SCF) convergence tolerance was set to $2.0 \times 10-6 \mathrm{eV} /$ atom. Valence electron configurations, Fe $3 \mathrm{~d}^{6} 4 \mathrm{~s}^{2}, \mathrm{~S} 3 \mathrm{~s}^{2} 3 \mathrm{p}^{6}$, and O $2 \mathrm{~s}^{2} 2 \mathrm{p}^{4}$, 
were calculated. For the calculation of the density of states, the value of smearing is $0.1 \mathrm{eV}$. All calculations were conducted in the reciprocal space with spin polarisation.

The adsorption energy of water molecules on the pyrite (100) surface was calculated as follows:

$$
E_{\text {ads }}=\frac{1}{n}\left(E_{n \mathrm{H}_{2} \mathrm{O} / \text { surface }}-n E_{\mathrm{H}_{2} \mathrm{O} / \text { surface }}-E_{\text {surface }}\right)
$$

where $E_{\text {ads }}$ represents the adsorption energy, $E_{n \mathrm{H} 2 \mathrm{O} / \text { surface }}$ represents the total energy of water molecules on the surface, and $E_{\mathrm{H} 2 \mathrm{O}}$ and $E_{\text {surface }}$ represent water molecules and the $3 \times 2$ supercell energy before adsorption, respectively. The lower the adsorption energy, the more stable the adsorption.

Molecular dynamics (MD) simulations using the Forcite module (Sun, 1998) were performed to investigate the effect of temperature on the adsorption of water molecules. The NVT ensemble and universal forcefield were used. The energy minimization model was chosen as the initial configuration of the molecular dynamics calculation. The periodic boundary condition was used in the dynamics simulation and the calculation was simulated with a time step of $1.0 \mathrm{fs}$ and total simulation time of 100.0 ps.

\section{Results and discussion}

\subsection{Adsorption of few water molecules}

The adsorption structures of $\mathrm{H}_{2} \mathrm{O}$ molecules at coverages of 1/12, 6/12, and 12/12 are shown in Fig. 1 . The adsorption of an isolated water molecule has been widely studied through experimental and theoretical methods (Stirling et al., 2003, Rosso et al., 1999, Li et al., 2018, Knipe et al., 1995, Pettenkofer et al., 1991, Tichomirowa and Junghans, 2009). In this study, an isolated water molecule is adsorbed onto the pyrite (100) surface with an adsorption energy of $-62.81 \mathrm{~kJ} / \mathrm{mol}$ and the length of the Fe-O bond is $2.096 \AA$. Our simulated results are consistent with other experimental and theoretical results. In addition, the two H atoms in water adsorb onto S sites with H-S hydrogen bond lengths of $2.578 \AA$ and $2.833 \AA$. This is consistent with the calculations by Sit et al. (2012). They calculated an adsorption energy of $-65.7 \mathrm{~kJ} / \mathrm{mol}$ for an isolated water molecule with a $2.13 \AA \mathrm{Ae}-\mathrm{O}$ bond length.

Figs. 1b-c show that as the water coverages increases, the Fe-O bond lengths increase (approximately $2.108 \AA$ at $6 / 12$ coverage and $2.125 \AA$ at $12 / 12$ coverage). At water coverage of $6 / 12$ (half-full coverage), there is no $\mathrm{H}-\mathrm{O}$ hydrogen bond formed due to the long distance between water molecules but there are $\mathrm{H}-\mathrm{O}$ hydrogen bonds formed in addition to the H-S hydrogen bonds at 12/12 (full coverage). The adsorption energy is calculated as $-57.29 \mathrm{~kJ} / \mathrm{mol}$ per water molecule at $6 / 12$ coverage and $-64.91 \mathrm{~kJ} / \mathrm{mol}$ per water molecule at 12/12 coverage. This result is not consistent with Sit et al. (2012) and Stirling et al. (2003) who suggested that the adsorption energy per water molecule is reduced at full coverage compared to at $1 / 8$ and $1 / 4$ coverage, respectively (their calculated model contains 8 and 4 Fe sites on the top surface) but is consistent with the result of de Leeuw et al. (2000) who suggested that the adsorption energy of the water monolayer is lower than that of an isolated water molecule on a planar (100) surface. They calculated the water-water interactions to be attractive at $-8.1 \mathrm{~kJ} / \mathrm{mol}$. Hence, they proposed that the main reason for the reduced adsorption energy of the full monolayer is not due to the repulsive interactions between the adsorbed water molecules themselves. Our simulation suggests that in addition to H-S bonds, H-O hydrogen bonds are formed at full coverage, enhancing the adsorption of water molecules. The water-water interaction can be clearly seen from Fig. 1c, where the H orientation is changed compared to that at water coverages of $1 / 12$ and 6/12. Stirling et al. (2003) proposed that this is because the interaction of $\mathrm{O}-\mathrm{H}$ is stronger than that of $\mathrm{S}-\mathrm{H}$.

It should also be noted that the bonding direction of Fe-O atoms is along the direction of the Fe-S bond in the sublayer (the next layer below the top layer), indicating that water molecules are directionally arranged on the pyrite surface. This phenomenon is consistent with the study by Philpott et al. (2004) By analysing the concentration distribution of oxygen and hydrogen atoms, they determined that the peak of the oxygen atoms surrounds the peak of the two hydrogen atoms, suggesting that the direction of the water molecule formation in this layer is certain.

\subsection{Adsorption of water molecules in excess of a monolayer}

The adsorption properties of $\mathrm{H}_{2} \mathrm{O}$ on the pyrite surface in excess of a monolayer were then studied and 


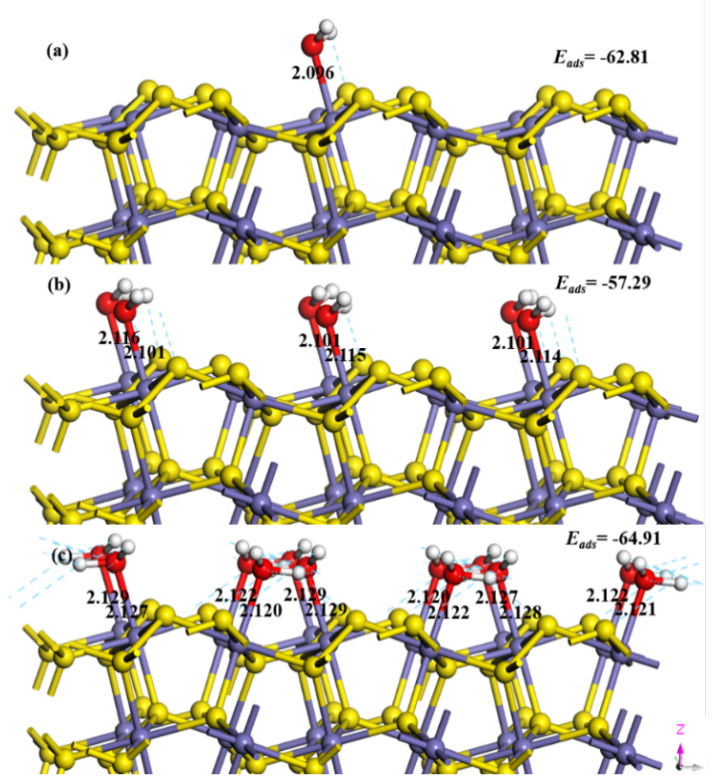

Fig. 1. Adsorption structures of $\mathrm{H}_{2} \mathrm{O}$ molecule at coverages of 1/12 (a), 6/12 (b) and 12/12 (c) of a monolayer. The blue dotted lines represent hydrogen bonds (including $\mathrm{H}-\mathrm{S}$ and $\mathrm{H}-\mathrm{O}$ )

Fig. 2 shows the adsorption structures of 2-6 layers of $\mathrm{H}_{2} \mathrm{O}$ molecules on the surface. The adsorption energies of two layers, three layers, and six layers of water molecules were calculated as $-46.43,-48.29$, and $-46.46 \mathrm{~kJ} / \mathrm{mol}$ per water molecule, respectively, which are less than that of a monolayer of water molecules $(-64.91 \mathrm{~kJ} / \mathrm{mol})$. These results indicate that the interaction between water molecules is weaker than the interaction between water molecules and the pyrite surface, resulting in reduced adsorption energy with increasing water molecules.

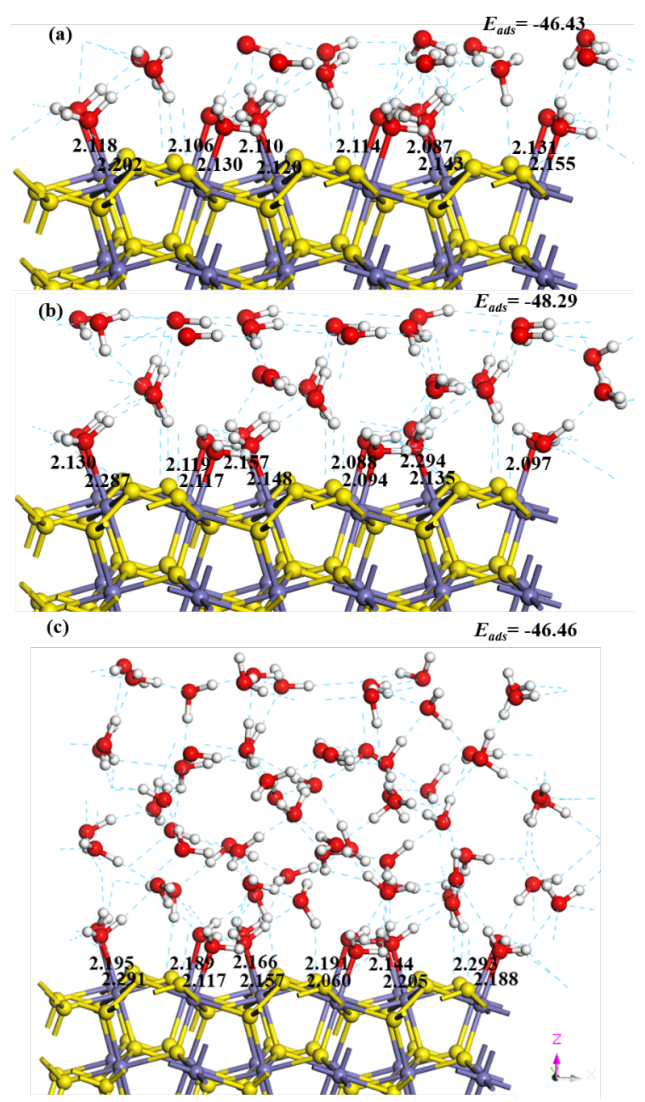

Fig. 2. Adsorption configuration of 2 layers of $\mathrm{H}_{2} \mathrm{O}$ molecule (a), 3 layers $\mathrm{H}_{2} \mathrm{O}$ of molecule (b), and 6 layers of $\mathrm{H}_{2} \mathrm{O}$ molecule (c) 
Finally, the vacuum was filled with $120 \mathrm{H}_{2} \mathrm{O}$ molecules (corresponding to 10 layers of $\mathrm{H}_{2} \mathrm{O}$ molecules), producing a water density of $1.03 \mathrm{~g} / \mathrm{cm}^{3}$. Fig. 3 shows the adsorption structure on the pyrite surface. According to the adsorption structure, the water molecules can be divided into three areas in the $\mathrm{z}$-direction (the vacuum thickness is $20.0 \AA$ ); the first area within $5.5 \AA$ above the surface (with a thickness of $5.5 \AA$ ), the second area from 5.5 to $11.0 \AA$ (with a thickness of $5.5 \AA$ ) above the surface, and the third area from 11.0 to $20 \AA$ (with a thickness of $9.0 \AA$ ) above the surface. It is shown that the arrangement of water molecules is most regular in the first area. This can be ascribed to the ordered adsorption of the first layer of water. The arrangement of water molecules in the second area also has some regularity but those in the third area are arranged haphazardly. These results suggest that the interaction between the pyrite surface and water molecules becomes weaker with increasing distance. This is consistent with Philpott et al. (2004), i.e., as the distance from the surface increases, it gradually decreases to the level of pure water density $\left(1 \mathrm{~g} / \mathrm{cm}^{3}\right)$.

The density of $\mathrm{H}_{2} \mathrm{O}$ molecules in different areas was calculated as shown in Fig. 4. It is demonstrated that the densities in the first, second and third area are 1.12, 1.08 and $0.95 \mathrm{~g} / \mathrm{m}^{3}$, respectively. This suggests that the water close to the surface is tightly bound together owing to the strong interaction with the surface. Interestingly, the adsorption of water molecules in the second area is also influenced, although they are far away from the surface. However, the water density in the third area is apparently lower than that in the former two areas. These results suggest that the interaction distance between water molecules and the pyrite surface can reach $10 \AA$.

In addition, the structure of the adsorbed $\mathrm{H}_{2} \mathrm{O}$ molecule was analysed in terms of the $\mathrm{H}$-O distance and $\mathrm{H}-\mathrm{O}-\mathrm{H}$ angle (average value of the first layer of 12 water molecules bonded with $\mathrm{Fe}$ ), as shown in Table 1. It is shown that $\mathrm{d}_{\mathrm{H}-\mathrm{O}}$, as well as $\mathrm{H}-\mathrm{O}-\mathrm{H}$ angle, are reduced after adsorbing onto the surface, compared to free $\mathrm{H}_{2} \mathrm{O}$. It is clearly shown from Table 1 that the water molecules are somehow stretched due to their hydrogen-bonding interactions.

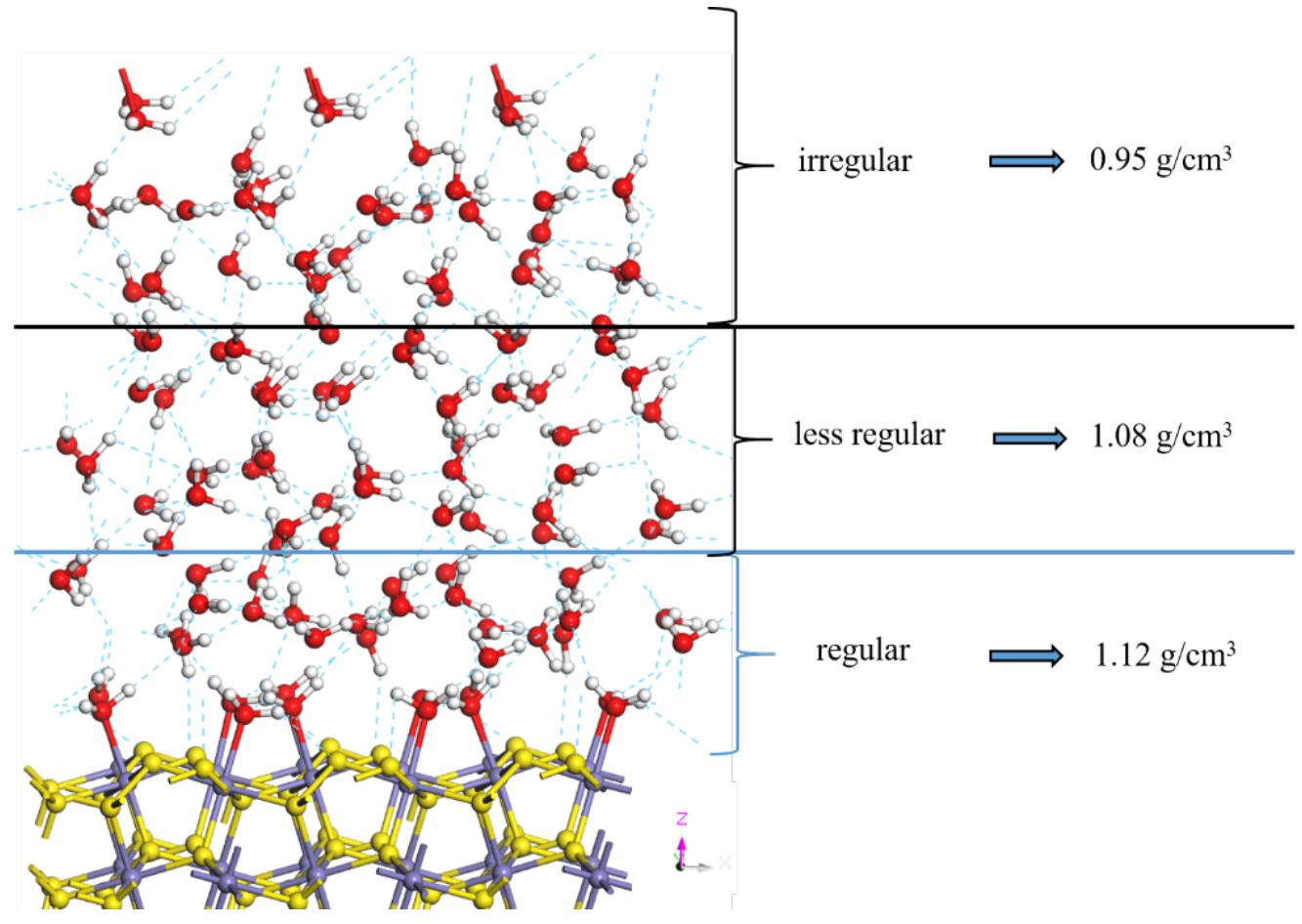

Fig. 3. Adsorption configuration of ten layers of water molecules

The structures of water molecules at coverages of $1 / 12$ and $6 / 12$ are very similar as the water is sufficiently dispersed with no interaction on the surface. With the increase in water coverage, there is a great fluctuation in bond length and angle for the first layer of the adsorbed water molecules. The $\mathrm{H}-\mathrm{O}$ bond length changes significantly compared to 1/12 coverage. In addition, the average H-O-H angle decreased. These results suggest that the structure of the first layer of the adsorbed water is affected by the continuous adsorption of water, mainly generated from the formation of $\mathrm{H}-\mathrm{O}$ hydrogen bonds 
between water molecules. This is consistent with the view of Stirling et al. (2003), that an additional water molecule will change the geometry structure of the first water monolayer.

Table 1. H-O bond length and bond angle of water molecules before and after adsorption onto the surface. $\mathrm{d}$ indicates bond length and $\mathrm{a}$ is an average value for the $\mathrm{H}-\mathrm{O}-\mathrm{H}$ angle

\begin{tabular}{ccc}
\hline Water coverage & $\mathrm{d}_{\mathrm{H}-\mathrm{O}} / \AA$ & $\mathrm{a}_{\mathrm{HOH}}{ }^{\circ}$ \\
\hline Theoretical value $^{\mathrm{a}}$ & 0.973 & 104.85 \\
${\text { Free } \mathrm{H}_{2} \mathrm{O} \text { molecule }}_{1 / 12}$ & 0.977 & 104.94 \\
$6 / 12$ & 0.985 & 106.27 \\
$12 / 12$ & $0.983-0.986$ & 106.29 \\
$24 / 12$ & $0.981-1.002$ & 106.22 \\
$36 / 12$ & $0.981-1.011$ & 106.44 \\
$72 / 12$ & $0.978-1.017$ & 104.95 \\
$120 / 12$ & $0.981-1.010$ & 104.49 \\
\hline
\end{tabular}

a Meng et al., 2004

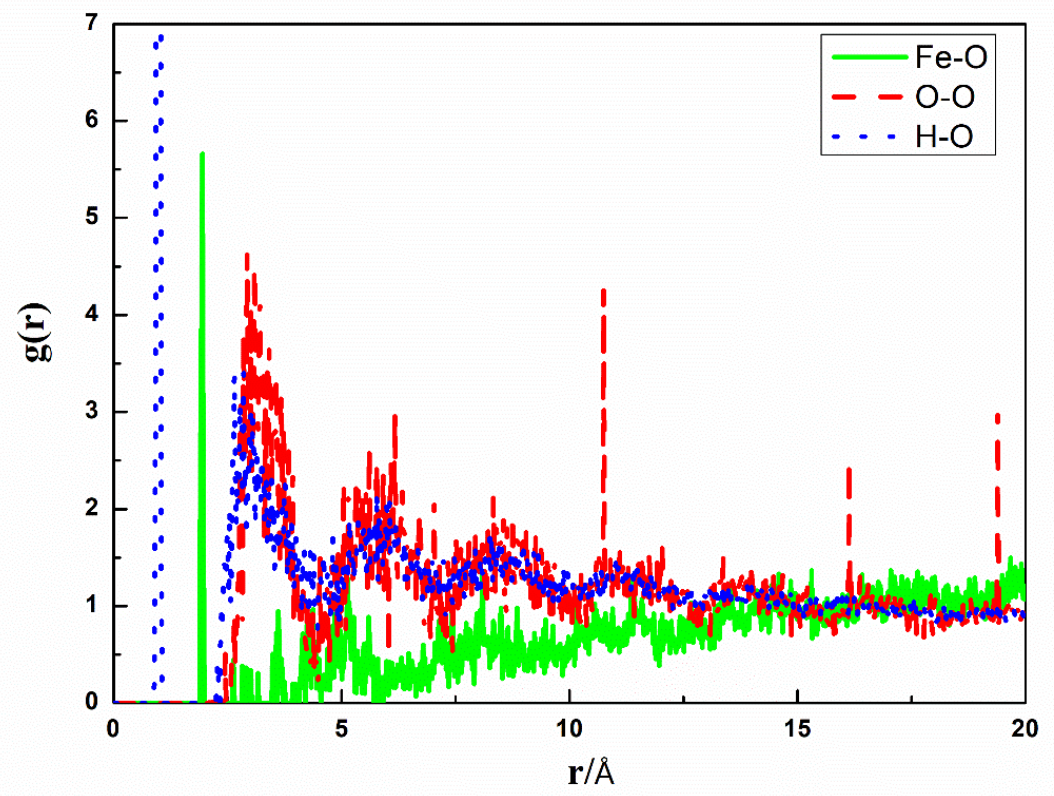

Fig. 4. Relative concentration profiles of $\mathrm{O}$ and $\mathrm{H}$ atoms of water molecules and between $\mathrm{O}$ atoms of water molecules and Fe atoms of pyrite surface, as a function of distance from the top pyrite (100) surface atoms at $298 \mathrm{~K}$

\subsection{MD simulations}

\subsubsection{Radial distribution function of water molecules}

The radial distribution function (Li et al., 2019, Liu et al., 2013) reflects the probability density between the two kinds of atoms and the aggregation characteristics of ions in the body. The radial distribution function reflects the atomic distribution characteristics and thermodynamic properties of the system. The radial distribution function can not only be used to study the order of matter but also to describe the correlation of electrons. The calculation formula is as follows (Zhang et al., 2018):

$$
g_{\alpha \beta}(r)=n_{\beta} / 4 \pi \cdot \rho_{\beta} \cdot r^{2} d r
$$

where $g_{\alpha \beta}(r)$ is the radial distribution function of $\beta, n_{\beta}$ is the number of $\beta$ ions around $\alpha$ in $r \rightarrow r+d_{r}$; $\rho_{\beta}$ is the number density of $\beta$ ions, and $\mathrm{r}$ is the distance between a ions and $\beta$ ions.

Fig. 4 shows the radial distribution functions of $\mathrm{O}-\mathrm{O}, \mathrm{O}-\mathrm{H}$, and $\mathrm{Fe}-\mathrm{O}$ at $298 \mathrm{~K}$. It is found that $\mathrm{gO}-\mathrm{O}$, $\mathrm{gO}-\mathrm{H}$ and $\mathrm{gFe}-\mathrm{O}$ are close to 1 when the water molecules are sufficiently far from the surface. According to the results of radial distribution functions, it is suggested that water molecules which are closest to 
the surface are adsorbed regularly. With the increase in the distance from the pyrite surface, the attenuation of the peak tends to be gentle and there is no obvious peak when the distance $r$ is more than $10 \AA$, indicating that the arrangement of water molecules far from the pyrite surface is disordered. Through the radial distribution functions of $\mathrm{Fe}-\mathrm{O}$, it is clearly illustrated that a water hydration structure is formed on the pyrite surface. The radial distribution of $\mathrm{O}$ and $\mathrm{O}$ atoms in water molecules at 273.15 K, $298.15 \mathrm{~K}, 310.15 \mathrm{~K}, 323.15 \mathrm{~K}, 353.15 \mathrm{~K}$, and $373.15 \mathrm{~K}$ is shown in Fig. 5. It was found that as the temperature increased, only the position of the first peak changed. However, the positions of the second, third, and fourth peaks are independent of temperature.

\subsubsection{Water molecules transport}

The relative concentration of water molecules along the z-axis is shown in Fig. 6. It is clearly found that the adsorption configuration of water molecules on the pyrite $\left(\begin{array}{lll}1 & 0 & 0\end{array}\right)$ surface along the z-axis is layer by layer. As is shown in Fig. 7, it is demonstrated that with the increase of temperature, the MSD of water molecules is increased. This result is consistent with the simulated result of Brunello et al. (2011) It can be seen clearly from Fig. 7 that MSD decreases as the amount of water molecules increases.

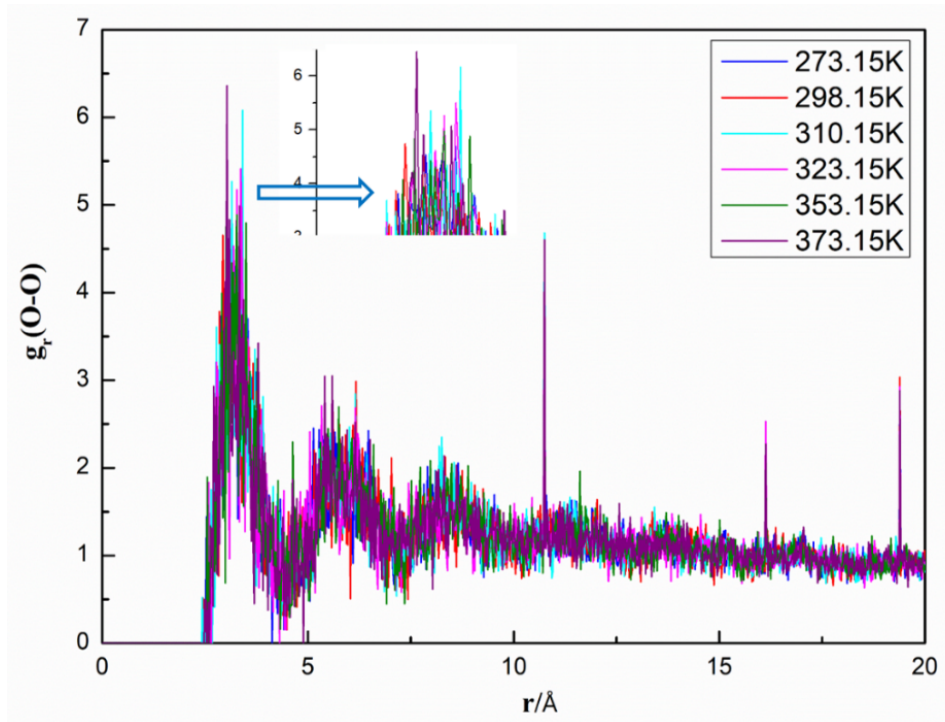

Fig. 5. Relative concentration profiles of the $\mathrm{O}$ and $\mathrm{O}$ atoms of water molecules as a function of the distance from the top pyrite (100) surface atoms at different temperature

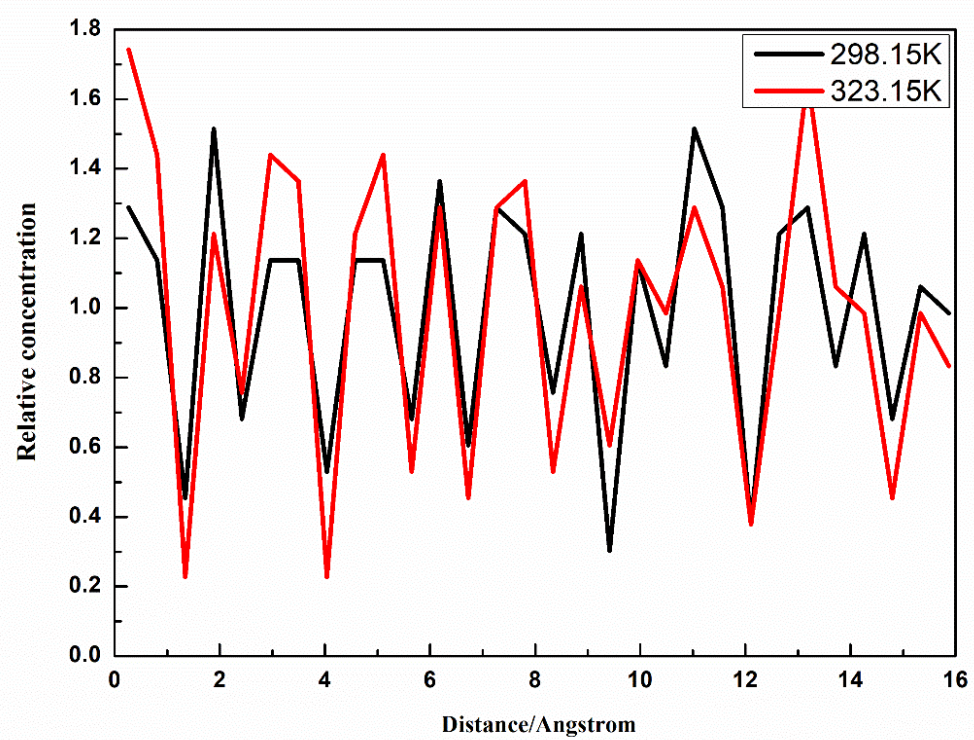

Fig. 6. Relative concentration of water molecules along $\left(\begin{array}{lll}1 & 0 & 0\end{array}\right)$ z-axis as a function of distance 


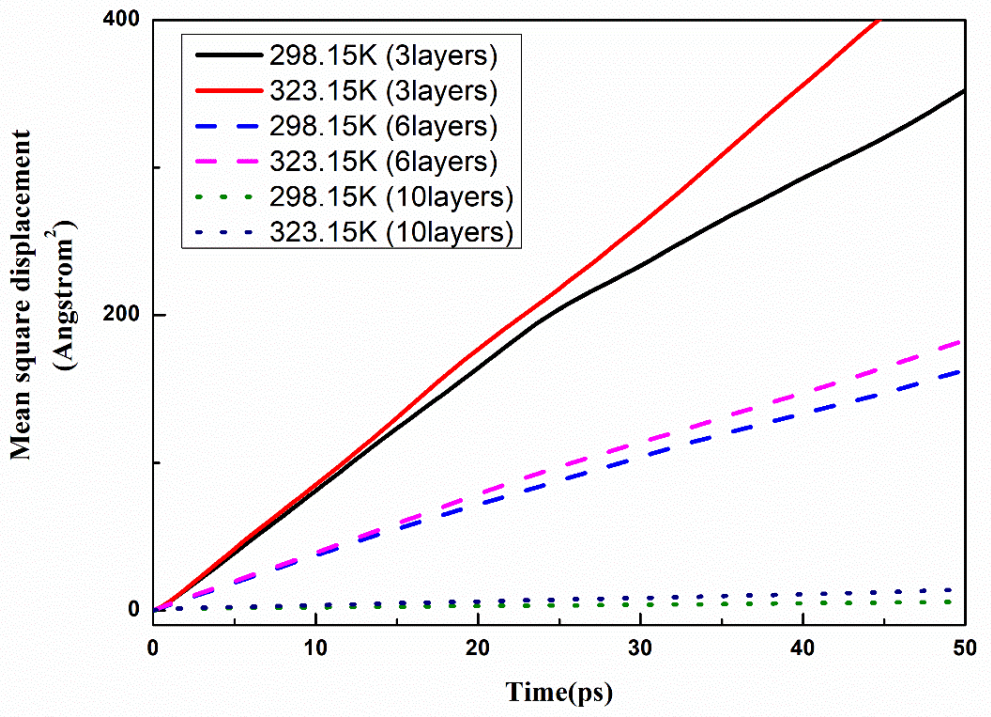

Fig. 7. Change of mean square displacement of water molecules as a function of time

\section{Conclusions}

Based on density functional theory (DFT), the adsorption structure of water molecules onto a pyrite surface has been simulated. When water molecules are adsorbed onto the pyrite surface, the first layer will be adsorbed along the direction of the Fe-S bond. $\mathrm{H}$ atoms in water molecules not only form a hydrogen bond with sulphur atoms on the pyrite surface but also form a hydrogen bond with $\mathrm{H}$ and $\mathrm{O}$ atoms in neighbouring water molecules. To simulate true water adsorption onto the pyrite surface, multilayer water molecules were simulated. The interaction between the pyrite surface and water molecules weakens with increasing distance. The analysis of molecular dynamics (MD) implies that water molecules are adsorbed onto the near surface in an orderly manner and water molecules far away from the pyrite (100) surface are disordered. The parts above the six layers are irregular. Through the radial distribution functions of $\mathrm{Fe}-\mathrm{O}$, it is found that a water hydration structure is formed on the pyrite surface. Then, the different coverage of water molecules may have an essential influence on the interaction between the pyrite surface and other reagents.

\section{Acknowledgements}

The authors are grateful for the financial support provided by the National Natural Science Foundation of China (NSFC) (grant numbers 51974094, 51764002, 51874106 and 51574092), and the Guangxi Natural Science Foundation (2017GXNSFAA198216 and 2018GXNSFAA281355).

\section{References}

BRUNELLO, G.F., MATEKER, W.R., LEE, S.G., CHOI, J.I., JANG, S.S., 2011. Effect of temperature on structure and water transport of hydrated sulfonated poly (ether ether ketone): A molecular dynamics simulation approach, Journal of Renewable and Sustainable Energy. 3(4), 043111-16.

CHADI, D.J., 1977. Special points for Brillouin-zone integrations, Physical Review B. 16(4), 1746-1747.

CHANDER, S., 1991. Electrochemistry of sulfide flotation: Growth characteristics of surface coatings and their properties, with special reference to chalcopyrite and pyrite, International Journal of Mineral Processing. 33(1-4), 121-134.

CHEN, J.H., LONG, X.H., CHEN., Y., 2014. Comparison of Multilayer Water Adsorption on the Hydrophobic Galena (PbS) and Hydrophilic Pyrite (FeS $S_{2}$ Surfaces: A DFT Study, The Journal of Physical Chemistry C. 118, 11657-11665.

CHENG, F.Q., CAO, Q.B., GUAN, Y.S., CHENG, H.G., WANG, X.M., MILLER, J.D., 2013. FTIR analysis of water structure and its influence on the flotation of arcanite $\left(\mathrm{K}_{2} \mathrm{SO}_{4}\right)$ and epsomite $\left(\mathrm{MgSO}_{4} \cdot 7 \mathrm{H}_{2} \mathrm{O}\right)$, International Journal of Mineral Processing. 122, 36-42.

CLARK, S.J., SEGALLII, M.D., PICKARDII, C.J., HASNIP, P.J., PROBERT, M.I.J., REFSON, K., PAYNE, M.C., 2005. First principles methods using CASTEP, Z. Kristallogr. 220, 567-570. 
CRISCENTI, L.J., HO, T.A., HART, D., 2018. Structural properties of aqueous solutions at the (100) and (101) goethite surfaces by molecular dynamics simulation, Langmuir. 34(48), 8b02612.

de LEEUW, N.H., PARKER, S.C., SITHOLE, H.M., NGOEPE, P.E., 2000. Modeling the surface structure and reactivity of pyrite: introducing a potential model for $\mathrm{FeS}_{2}$, Journal of Physical Chemistry B. 104(33), 7969-7976.

FENG, B., ZHONG, C.H., ZHANG, L.Z., GUO, Y.T., WANG, T., HUANG, Z.Q., 2020. Effect of surface oxidation on the depression of sphalerite by locust bean gum, Minerals Engineering. 146, 106142.

GUEVREMONT, J.M., BEBIE, J., ELSETINOW, A.R., STRONGIN, D.R., SCHOONEN, M.A.A., 1998. Reactivity of the (100) plane of pyrite in oxidizing gaseous and aqueous environments: effects of surface imperfections, Environmental Science \& Technology. 32(23), 1453-1457.

JIN J.Q., 2016. Wetting and interfacial water analysis of selected mineral surfaces as determined by molecular dynamics simulation and sum frequency vibrational spectroscopy, University of Utah.

JIN, J.Q., MILLER, J.D., DANG, L.X., WICK, C.D., 2015. Effect of surface oxidation on interfacial water structure at a pyrite (100) surface as studied by molecular dynamics simulation, International Journal of Mineral Processing. 139, 64-76.

KNIPE, S.W., MYCROFT, J.R., PRATT, A.R., NESBITT, H.W., BANCROF., G.M., 1995. X-ray photoelectron spectroscopic study of water adsorption on iron sulphide minerals, Geochimica et Cosmochimica Acta. 59(6), 10791090.

LI, Y.Q., CHEN, J.H., CHEN, Y., ZHAO, C.H., ZHANG, Y.B., KE., B.L., 2018. Interactions of Oxygen and Water Molecules with Pyrite Surface: A New Insight, Langmuir. 34, 1941-1952.

LI, Y.Q., CHEN, J.H., CHEN, Y., ZHU, Y.G., LIU, Y.C., 2019. DFT Simulation on Interaction of $\mathrm{H}_{2} \mathrm{O}$ Molecules with ZnS and $\mathrm{Cu}$-Activated Surfaces, The Journal of Physical Chemistry C. 123, 3048-3057.

LIU, X.W., ZHU, R.L., MA, J.F., GEI, F., XU, Y., LIU, Y., 2013. Molecular dynamics simulation study of benzene adsorption to montmorillonite: Influence of the hydration status, Colloids and Surfaces A-Physicochemical and Engineering Aspects. 434(19), 200-206.

MENG, S., WANG, E.G., GAO, S.W., 2004. Water adsorption on metal surfaces: A general picture from density functional theory studies, Physical Review B, Condensed Matter. 69(19), 195404:1-13.

NESBITT, H.W., MUIR., I.J., 1994. X-ray photoelectron spectroscopic study of a pristine pyrite surface reacted with water vapor and air, Geochimica et Cosmochimica Acta. 58(21), 4667-4679.

PERDEW, J.P., CHEVARY, J.A., VOSKO, S.H., JACKSON, K.A., PEDERSON, M.R., SINGH, D.J., FIOLHAIS, C., 1993. Erratum: Atoms, molecules, solids, and surfaces: Applications of the generalized gradient approximation for exchange and correlation, Physical Review B. 48(7), 4978-4978.

PERDEW, J.P., WANG, Y., 1992. Accurate and simple analytic representation of the electron-gas correlation energy, Physical Review B. 45(23), 13244-13249.

PETTENKOFER, C., JAEGERMANN, W., BRONOLD, M., 1991. Site specific surface interaction of electron donors and acceptors on $\mathrm{FeS}_{2}$ (100) cleavage planes, Berichte Der Bunsengesellschaft Für Physikalische Chemie. 95, 560-565.

PHILPOTT, M.R., GOLINEY, I.Y., LIN, T.T., 2004. Molecular dynamics simulation of water in a contact with an iron pyrite $\mathrm{FeS}_{2}$ surface, Journal of Chemical Physics. 120(4), 1943-1950.

PRINCE, K.C., MATTEUCCI, M., KUEPPER, K., CHIUZBAIAN, S.G., BARTKOWSKI, S., NEUMANN, M., 2005. Core-level spectroscopic study of $\mathrm{FeO}$ and $\mathrm{FeS}_{2}$, Physical Review B. 71, 0851021-0851029.

ROSSO, K.M., BECKER, U., HOCHELLA, M.F., 1999. The interaction of pyrite $\{100\}$ surfaces with $\mathrm{O}_{2}$ and $\mathrm{H}_{2} \mathrm{O}$ : Fundamental oxidation mechanisms, American Mineralogist. 84(10), 1549-1561.

SANDRINA, M., UTA., M., 2010. Investigation of the water-pyrite-(100)-interface with GIXRD, Acta Crystallographica. 66(a1), s98-s99.

SEGALL, M.D., LINDAN, P.J.D., PROBERT, M.J., PICKARD, C.J., HASNIP, P.J., CLARK S.J., PAYNE, M.C., 2002. First-principles simulation: ideas, illustrations and the CASTEP code, Journal of Physics Condensed Matter. 14(11), 2717-2744.

SIT, H.L., COHEN, M.H., SELLONI, A., 2012. Interaction of Oxygen and Water with the (100) Surface of Pyrite: Mechanism of Sulfur Oxidation, Journal of Physical Chemistry Letters. 3(17), 2409-2414.

STIRLING, A., BERNASCONI, M., PARRINELLO, M., 2003. Ab initio simulation of water interaction with the (100) surface of pyrite, Journal of Chemical Physics. 118(19), 8917-8926.

SUN, J.H., 1998. COMPASS: An ab Initio Force-Field Optimized for Condensed-Phase Applications Overview with Details on Alkane and Benzene Compounds, Journal of Physical Chemistry B. 102, 7338-7364.

TAUSON, V.L., BABKIN, D.N., LUSTENBERG, E.E., LIPKO, S.V., PARKHOMENKO, I.YU., 2008. Surface 
typochemistry of hydrothermal pyrite: Electron spectroscopic and scanning probe microscopic data. I. Synthetic pyrite, Geochemistry International. 46(6), 565-577.

TICHOMIROWA, M., JUNGHANS, M., 2009. Oxygen isotope evidence for sorption of molecular oxygen to pyrite surface sites and incorporation into sulfate in oxidation experiments, Applied Geochemistry. 24(11), 2072-2092.

VANDERBILT, D., 1990. Soft self-consistent pseudopotentials in a generalized eigenvalue formalism, Physical Review B. 41, 7892-7895.

XI, P., SHI, C.X., YAN, P.K., LIU, W.L., TANG. L.G., 2019. DFT study on the influence of sulfur on the hydrophobicity of pyrite surfaces in the process of oxidation, Applied Surface Science. 466, 964-969.

ZHANG, Y.Y., CHEN, M., DENG, Y., JIN, Y., LU, Y.H., XIA, Y., 2018. Molecular Dynamics Simulation of Temperature and Pressure Effects on Hydration Characteristics of Montmorillonites, Journal of the Chinese Ceramic Society. 46(10), 1489-1498. 